\title{
Undiagnosed Obstructive Sleep Apnea and Postoperative Outcomes: A Prospective Observational Study
}

\author{
Uma Devaraj $^{\mathrm{a}}$ Srinivas Rajagopala $^{\mathrm{b}}$ Ajay Kumar $^{\mathrm{a}}$ Priya Ramachandran $^{\mathrm{a}}$ \\ Philip J. Devereaux ${ }^{c}$ George A. D'Souza ${ }^{a}$ \\ a Department of Pulmonary Medicine, St. John's Medical College Hospital, Bangalore, and bPSG Institute of \\ Pulmonary Medicine, PSG IMS\&R, Coimbatore, India; ' Departments of Clinical Epidemiology and Biostatistics and \\ Medicine, McMaster University, and the Population Health Research Institute, Hamilton Health Sciences, \\ Hamilton, ON, Canada
}

\section{Keywords}

Obstructive sleep apnea · Postoperative complications . Sleep study

\begin{abstract}
Background: The prevalence of undiagnosed obstructive sleep apnea (OSA) during preoperative evaluation and the best method to screen OSA and its association with postoperative complications remain unclear. Objectives: To determine the prevalence of undiagnosed OSA in preoperative Indian patients undergoing noncardiac surgery, to compare the diagnostic accuracy of the STOP-BANG questionnaire to a preoperative level III sleep study, and to assess the association of OSA with postoperative complications. Methods: A prospective cohort of 245 consecutive adults with $\geq 2$ risk factors for OSA who underwent noncardiac surgery between July 2011 and February 2013 were studied. The STOP-BANG questionnaire was administered to all patients, and 182/245 (74.2\%) patients underwent a preoperative level III sleep study. Patients were followed for postoperative complications in hospital and contacted at 30 days after surgery. Results: 70/182 (38.5\%) obtained a new diagnosis of OSA, including 11/182 (6\%) with moderate to severe OSA (apneahypopnea index $\geq 15 / h$ ). On logistic regression analyses, the
\end{abstract}

\section{KARGER}

(C) 2017 S. Karger AG, Basel

E-Mail karger@karger.com

www.karger.com/res presence of OSA was independently associated with postoperative oxygen desaturation (OR 5.96, 95\% Cl 2.35-15.1, $p<$ 0.01 ), a postoperative complication within 7 days (OR 3.63, $95 \% \mathrm{Cl} 1.77-7.45, p<0.01$ ) and within 30 days (OR 3.5, 95\% $\mathrm{Cl} 1.74-7.1, p<0.01)$. The STOP-BANG questionnaire did not identify $12 / 70$ (17\%) of the patients diagnosed with OSA and classified $28 \%$ of the cohort as OSA when the level III sleep study was negative. Conclusions: Unrecognized OSA is common in preoperative patients and is independently associated with postoperative complications. The STOP-BANG questionnaire had a lower performance in the diagnosis of OSA in a South Indian population than the level III sleep study.

(c) 2017 S. Karger AG, Basel

\section{Introduction}

Major surgeries are frequently performed worldwide, with an estimated 235 million procedures per year, and major postoperative respiratory and cardiovascular complications occur in $10-15 \%$ of this population [1]. Obstructive sleep apnea (OSA) is common, with a prevalence of $2-4 \%$ in unselected adult populations $[2,3]$. Previous studies have reported a $3-24 \%$ prevalence of

Dr. Uma Devaraj

3rd floor, PFT Lab, Oncology Block, St. John's Medical College Sarjapur Road

Bangalore 560034 (India)

E-Mail druma.devaraj@gmail.com 
unrecognized OSA in preoperative adult surgical populations [4-6]. While the STOP-BANG questionnaire has been validated for preoperative screening of OSA, age, $\mathrm{BMI}$, and neck circumference cutoffs in South Indians are likely quite different from the original cohort and the effect of this on the performance of the screening test is unknown.

In the perioperative period, the rapid eye movement (REM) suppression of opioids, compounded by the effects of sedatives and anesthetic agents on upper airway tone and arousal response, together with the supine posture, can worsen OSA. These events are magnified by the effects of sleep deprivation, sleep fragmentation, and postoperative REM sleep rebound [7]. Nocturnal electrocardiographic changes of ischemia have been reported in patients with OSA, even in the absence of significant coronary artery disease [8]. Greater rates of postoperative oxygen desaturation, delirium, myocardial infarction, arrhythmias, stroke, and wound breakdown are linked to postoperative REM rebound and sympathetic activation due to OSA [9-11].

Despite the global magnitude of this perioperative problem, several basic questions regarding OSA in Indian patients have not been adequately addressed. We therefore undertook a cohort study of patients undergoing noncardiac surgery in a large tertiary care hospital in South India to assess the prevalence of unrecognized OSA, the diagnostic accuracy of the STOP-BANG questionnaire, and the relationship between OSA and perioperative respiratory and cardiovascular complications.

\section{Materials and Methods}

This study was conducted in a large tertiary care hospital in South India between July 2011 and February 2013. All adults undergoing elective noncardiac surgery were identified by the preoperative surgical list obtained from the department of surgery a day prior to the surgery. Consecutive patients were assessed for eligibility criteria (Fig. 1). The study was initiated after approval from the institutional ethics committee and after obtaining written informed consent from the study participants.

\section{Study Subjects}

Patients had to fulfil the following inclusion criteria: $\geq 18$ years of age, $\geq 2$ risk factors being positive using the STOP-BANG questionnaire [12] (i.e., history of snoring, history of feeling tired or sleepy during daytime, history of witnessed apneas during sleep, history of hypertension, BMI $>28$ [lower BMI taken as recommended by the WHO and NICE guidelines for BMI in Asians] [13, 14 ], and neck circumference $>40 \mathrm{~cm}$ [online suppl. Appendix 1.3; see www.karger.com/doi/10.1159/000470914 for all online suppl. material]), and undergoing elective noncardiac surgery under gen- eral anesthesia (Fig. 1; online suppl. Appendix 1.1). Exclusion criteria included a prior diagnosis of OSA, preoperative pneumonia, obstructive lung disease, chronic respiratory failure, outpatient surgery, the concurrent administration of sedatives, and lack of patient or physician consent.

\section{Preoperative Assessment}

All eligible patients underwent a standardized measurement of the BMI and neck circumference (online suppl. Appendix 1.1). All patients enrolled in the study were scored on the STOP-BANG questionnaire in English or in a validated local language. The study personnel used a standardized data collection form to record preoperative and intraoperative demographic variables. Patients were offered a level III sleep study (Resmed Apnea-link Plus ${ }^{\mathrm{TM}}$ ) on the night prior to surgery. This device uses noninvasive flow monitoring, pulse oximetry, and thoracic and abdominal bands to define airflow obstruction by the absence of flow despite continuing ventilatory effort. The level III sleep study is widely used as a screening test for OSA prior to polysomnography referral and has a sensitivity of at least $85 \%$ for any OSA and nearly $100 \%$ for moderate to severe OSA. The level III sleep study has good diagnostic performance when compared with level I sleep tests in adult patients with a high pretest probability of OSA [15].

Patients, health care providers, data collectors, and outcome adjudicators were blinded to the results of the sleep study until the patient had completed the study. All the sleep studies were scored by a single sleep technologist and reviewed by a physician certified in sleep medicine (U.D.).

All preoperative anxiolytic or sedative agents were withheld. All enrolled patients underwent continuous pulse oximetry monitoring for $24 \mathrm{~h}$ following surgery and a chest radiograph at $72 \mathrm{~h}$. All enrolled patients were followed throughout their hospitalization, and study personnel phoned patients at 30 days after surgery. If this fell on a weekend or holiday, the next nearest working day was used to complete the interview. A single interviewer unaware of the results of the overnight sleep study (A.K.) was responsible for all the interviews. Each interview lasted approximately $10 \mathrm{~min}$. Any occurrence of predefined postoperative complications (PCs) was confirmed by the in-patient records. All patients with moderate to severe OSA on the screening sleep study were subsequently contacted after the 30-day follow-up had been completed and advised a follow-up with the pulmonologist in our hospital. Similarly, patients with a STOP-BANG score $\geq 3$ but negative for OSA in the level III sleep study were also advised a follow-up with the pulmonologist.

\section{Outcome Measures}

The presence of OSA was defined by an apnea-hypopnea index (AHI) of $\geq 5$ on the sleep study. Apnea was defined as per the AASM Scoring Manual (version 2.0.2; there was a drop in the peak signal excursion by $\geq 90 \%$ of the pre-event baseline using an oronasal thermal sensor, and this signal drop lasted for $\geq 10 \mathrm{~s}$ ). Hypopnea was defined as a peak signal excursion drop by $\geq 30 \%$ of the pre-event baseline and the duration of this signal drop lasted for $\geq 10$ s along with $\geq 3 \%$ oxygen desaturation from the pre-event baseline. The oxygen desaturation index (ODI) was defined as the average number of desaturation $\geq 4 \%$ per hour.

PCs were defined as the development of any predefined complication at hospital discharge, i.e. respiratory PCs (a composite outcome of pneumonia, sepsis [respiratory and nonrespiratory],

OSA and Postoperative Outcomes 


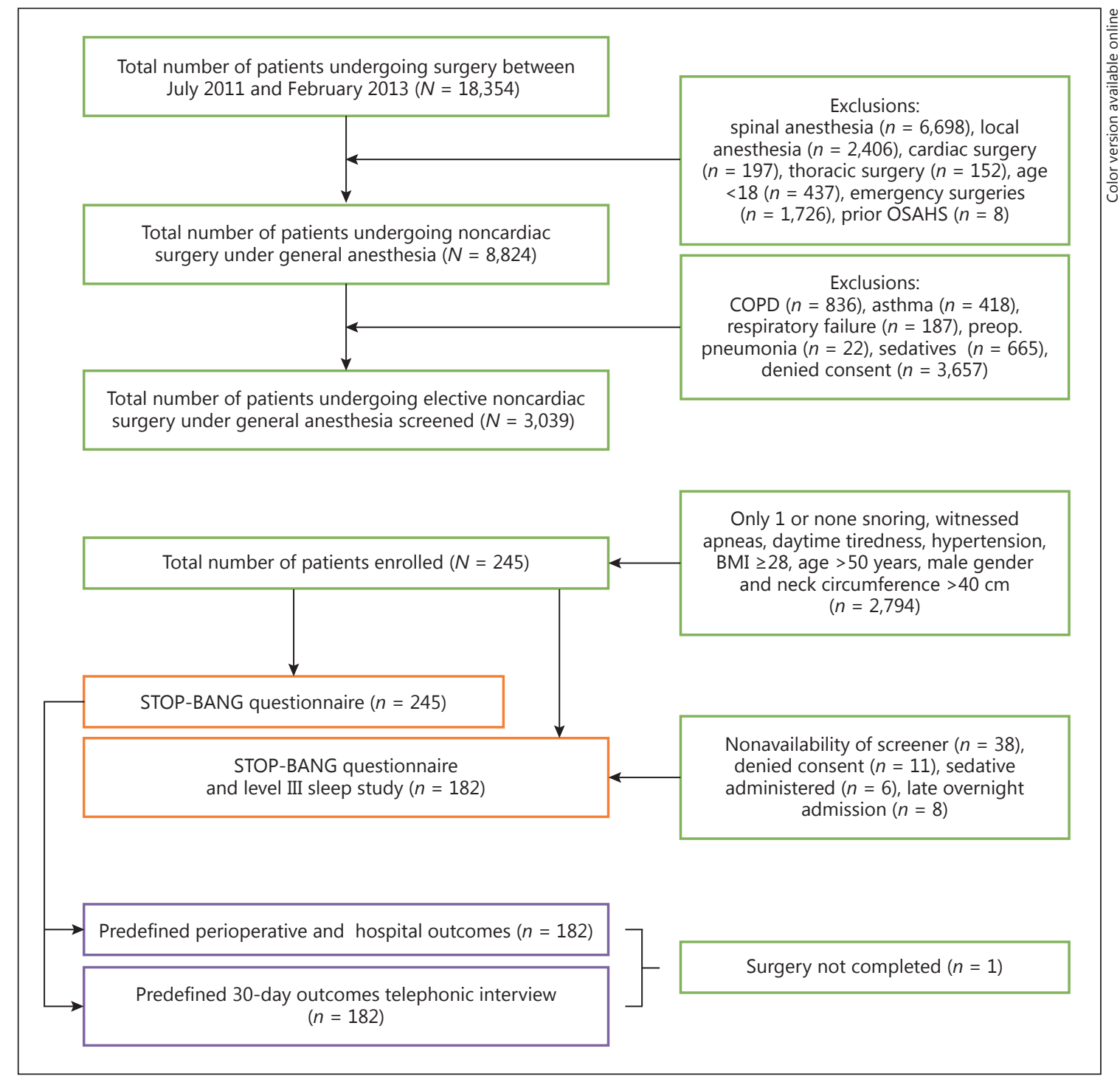

Fig. 1. Flowchart of patients screened and enrolled in a study of obstructive sleep apnea and its association with postoperative complications. OSAHS, obstructive sleep apnea hypopnea syndrome; COPD, chronic obstructive pulmonary disease.

postoperative oxygen desaturation or respiratory failure, defined as (a) persistent hypoxemia: arterial oxygen saturation lower than $85 \%$ despite the use of $\mathrm{FiO}_{2}$ of 0.5 for $>10 \mathrm{~min}$ and (b) respiratory acidosis [arterial $\mathrm{pH}$ level $<7.30$ with $\mathrm{PaCO}_{2}>50 \mathrm{~mm} \mathrm{Hg}$ ]) and cardiovascular PCs (a composite outcome of myocardial infarction, congestive cardiac failure, stroke, or arrhythmias) (online suppl. Appendix 1.1).

\section{Statistical Analysis}

We anticipated a $25 \%$ prevalence of undiagnosed OSA in the preoperative noncardiac surgery population with at least 2 risk factors for OSA. Using published data of $25 \%$ PCs in patients with OSA and 5\% PCs in patients without OSA postoperatively, we estimated a sample size of 132 at $80 \%$ power and at a $5 \%$ a level
(N Master version 1.1). Using a 20\% refusal rate for an overnight preoperative sleep study and a $10 \%$ attrition rate during follow-up, the planned sample size was 175 patients. Continuous variables are reported as mean \pm SD and discrete variables as frequency proportions. Comparison for continuous variables was done by the Student $t$ test or Wilcoxon rank sum where appropriate, and for proportions by the $\chi^{2}$ or the Fisher exact test where appropriate. We undertook multivariable logistic regression and the dependent variable was the development of the predefined PCs and the potential independent variables included the presence of any OSA, age, ASA (American Society of Anesthesiologists) risk class, general anesthesia duration, comorbidities and type of surgery. Analysis was done using statistical software STATA/IC version 12.1. A two-tailed $p$ value of $<0.05$ was considered statistically significant. 
Table 1. Demographic characteristics of patients with and without OSA based on their sleep study

\begin{tabular}{lccc}
\hline Parameters & \multicolumn{2}{l}{ OSA } & \multirow{2}{*}{$p$ value } \\
\cline { 2 - 3 } & $\begin{array}{l}\text { present } \\
(n=70 / 182)\end{array}$ & $\begin{array}{c}\text { absent } \\
(n=112 / 182)\end{array}$ & 0.005 \\
\hline Age, years & $52.68 \pm 11.48$ & $46.55 \pm 15.51$ & 0.21 \\
Gender (male/total) & $36 / 70(51.4 \%)$ & $47 / 112(41.9 \%)$ & 0.57 \\
History of snoring & $58 / 70(82.8 \%)$ & $89 / 112(79.5 \%)$ & 0.95 \\
History of excessive sleepiness & $11 / 70(15.7 \%)$ & $18 / 112(16.1 \%)$ & 0.01 \\
History of witnessed apnea & $7 / 70(0.1 \%)$ & $2 / 112(0.02 \%)$ & 0.02 \\
Diagnosed with arterial hypertension & $36 / 70(51.4 \%)$ & $38 / 112(33.9 \%)$ & 0.40 \\
Height, cm & $160.34 \pm 8.19$ & $161.45 \pm 8.89$ & $<0.001$ \\
Weight, kg & $70.68 \pm 14.25$ & $63.32 \pm 10.92$ & $<0.001$ \\
Body mass index & $27.24 \pm 5.00$ & $24.09 \pm 4.02$ & $<0.001$ \\
Neck circumference, cm & $37.09 \pm 4.22$ & $34.83 \pm 3.72$ & $<0.001$ \\
Waist circumference, cm & $94.06 \pm 12.37$ & $87.22 \pm 10.31$ & 0.003 \\
Hip circumference, cm & $104.21 \pm 13.15$ & $98.83 \pm 11.07$ & 0.085 \\
Waist/hip ratio & $0.90 \pm 0.07$ & $0.88 \pm 0.08$ & $<0.001$ \\
STOP-BANG score $\geq 3$ present & $58 / 70$ & $39 / 112$ & 0.44 \\
ASA class $\geq 3$ & $8 / 70$ & $9 / 112$ & 0.22 \\
Type of surgery (general surgery/others) & $33 / 70$ & $58 / 112$ & 0.06 \\
General anesthesia duration, $h$ & $3.27 \pm 1.9$ & $2.77 \pm 1.6$ & $<0.001$ \\
Apnea-hypopnea index/h & $12.3 \pm 13.03$ & $1.9 \pm 1.3$ & $<0.001$ \\
Median oxygen-desaturation index/h & $8(7.25)^{\mathrm{a}}$ & $2(3)^{\mathrm{a}}$ & \\
\hline
\end{tabular}

Figures are means \pm SD or numbers with percentages in parentheses, unless indicated otherwise. OSA, obstructive sleep apnea; STOP-BANG, consists of snoring, tired, fatigued, or sleepy, stopping breathing, pressure (hypertension), body mass index $\geq 35$, neck circumference $>40 \mathrm{~cm}$, and male gender; ASA, American Society of Anesthesiologists score.

${ }^{\text {a }}$ Interquartile range in parentheses.

Ethics

The study was approved by the Research Ethics Committee of St. John's Medical Hospital. All data abstracted were anonymized, thus ensuring confidentiality and patient privacy.

\section{Results}

Between July 2011 and February 2013, 8,824 patients underwent elective surgery under general anesthesia (Fig. 1), of whom 245 patients were enrolled. Before surgery, all included patients completed the STOP-BANG questionnaire; 182 of 245 patients (74\%) also underwent an overnight level III sleep study. The majority of patients (181 of 182 ; $99.5 \%)$ completed the scheduled surgery under general anesthesia on the day following the sleep study. All patients were followed up in hospital and by telephone on day 30 after surgery (Fig. 1).

The sleep study indicated that 70 of 182 patients (38.5\%) had undiagnosed OSA, including 11 of 182 patients $(6 \%)$ with moderate to severe OSA (AHI $\geq 15 / \mathrm{h}$ ).
Patients with OSA were more likely to be older, have diagnosed or treated hypertension and have a higher BMI, neck circumference, and waist-hip ratio (Table 1). The mean age of the OSA cohort was $52.7 \pm 11.5$ years. A higher percentage of undiagnosed OSA patients had a neck circumference $>40 \mathrm{~cm}(13 / 70$ [18.6\%]) as compared to patients who did not have OSA (5/107 [4.7\%]; OR 4.8, 95\% CI 1.5-16.6, $p<0.002)$; however, only 18/182 (9.9\%) had a neck circumference $>40 \mathrm{~cm}$. With a BMI $>35,5 / 70$ (7.1\%) versus $3 / 112(2.7 \%)$ had a diagnosis of OSA (OR $2.79, p=0.26)$; however, only $8 / 182(4.4 \%)$ of the cohort had a $\mathrm{BMI}>35$.

Among patients with OSA, 58 of 70 patients (88\%) had at least 3 risk factors identified on the STOP-BANG questionnaire. The STOP-BANG questionnaire misclassified $51 / 182(28 \%)$ patients with OSA and had a sensitivity of $82.8 \%$ (95\% CI $71.9-90.8$ ), a specificity of $65.2 \%$ (95\% CI 55.6-73.9), the positive likelihood ratio was 2.38 and the negative likelihood ratio was 0.26 when compared to the level III sleep study. 
Table 2. Outcomes of patients without OSA (AHI $<5 / \mathrm{h})$ compared with patients with mild OSA (AHI 5-15/h) and moderate to severe OSA $(\mathrm{AHI}>15 / \mathrm{h})$

\begin{tabular}{|c|c|c|c|c|}
\hline \multirow[t]{2}{*}{ Parameters } & \multirow{2}{*}{$\begin{array}{l}\text { No OSA } \\
(n=112 / 182, \\
60.9 \%)\end{array}$} & \multicolumn{2}{|l|}{ OSA } & \multirow[t]{2}{*}{$p$ value } \\
\hline & & $\begin{array}{l}\text { mild } \\
(n=59 / 182, \\
32.1 \%)\end{array}$ & $\begin{array}{l}\text { moderate to severe } \\
(n=11 / 182 \\
6 \%)\end{array}$ & \\
\hline Any complication at 7 days & $16 / 112(14.3)$ & $17 / 59(28.8)$ & $10 / 11(90.9)$ & $<0.001$ \\
\hline Combined respiratory complications ${ }^{\mathrm{a}}$ at 7 days & $9 / 112(8.0)$ & $5 / 59(8.5)$ & $5 / 11(45.4)$ & $<0.001$ \\
\hline Pneumonia or sepsis at 7 days & $7 / 112(6.25)$ & $5 / 59(8.5)$ & $3 / 11(27.3)$ & 0.05 \\
\hline Respiratory failure at 7 days & $4 / 112(3.5)$ & $1 / 59(1.7)$ & $5 / 11(45.4)$ & $<0.001$ \\
\hline Combined cerebrovascular events ${ }^{\mathrm{b}}$ at 7 days & $3 / 112(2.7)$ & $3 / 59(5.1)$ & $3 / 11(27.3)$ & 0.002 \\
\hline Myocardial infarction or congestive cardiac failure at 7 days & $3 / 112(2.7)$ & $3 / 59(5.1)$ & $3 / 11(27.3)$ & 0.002 \\
\hline Postoperative oxygen desaturation & $7 / 112(6.3)$ & $12 / 59(20.3)$ & $10 / 11(90.9)$ & $<0.001$ \\
\hline Pleural effusion at 7 days & $0 / 112(0)$ & $3 / 59(5.1)$ & $1 / 11(9.1)$ & 0.03 \\
\hline Any complication at 30 days & $18 / 112(16.1)$ & $20 / 59(33.8)$ & $10 / 11(90.9)$ & $<0.001$ \\
\hline Combined respiratory complications ${ }^{\mathrm{a}}$ at 30 days & $11 / 112(9.8)$ & $11 / 59(18.6)$ & $5 / 11(41.6)$ & 0.004 \\
\hline Pneumonia or sepsis at 30 days & $9 / 112(8.0)$ & $9 / 59(15.2)$ & $4 / 11(36.4)$ & 0.01 \\
\hline Respiratory failure at 30 days & $4 / 112(3.6)$ & $2 / 59(3.4)$ & $5 / 11(41.6)$ & $<0.001$ \\
\hline Combined cardio- and cerebrovascular events ${ }^{\mathrm{b}}$ at 30 days & $5 / 112(4.5)$ & $4 / 59(6.8)$ & $3 / 11(27.3)$ & 0.01 \\
\hline Myocardial infarction or congestive cardiac failure at 30 days & $5 / 112(4.5)$ & $4 / 59(6.8)$ & $3 / 11(27.3)$ & 0.01 \\
\hline Stroke at 30 days & $0 / 112(0)$ & $0 / 59(0)$ & $0 / 11(0)$ & n.a. \\
\hline Acute kidney injury at 30 days & $0 / 112(0)$ & $0 / 59(0)$ & $1 / 11(9.1)$ & n.a. \\
\hline Hospital duration (mean \pm SD), days & $5 \pm 5.4$ & $6.2 \pm 6.8$ & $9.7 \pm 9.4$ & 0.04 \\
\hline
\end{tabular}

Figures in parentheses are percentages. n.a., not available.

${ }^{a}$ The combined endpoint of pneumonia or sepsis or respiratory failure at 7 days. ${ }^{b}$ The combined endpoint of postoperative myocardial infarction or congestive cardiac failure or new-onset arrhythmias or postoperative stroke.

On exploratory ROC analysis using our data as a derivation dataset, a modified STOP-BANG questionnaire with an age cutoff of 40 years, a BMI of 28 and a neck circumference of $36 \mathrm{~cm}[16,17]$ was used to test the performance of the questionnaire. Sensitivity improved to $90 \%$ (95\% CI 80.5-95.9) with a loss of specificity (18.8\% [95\% CI 12-27.2]) when compared to the level III sleep study. No patients with moderate to severe OSA (AHI $\geq 15 / \mathrm{h}$ ) were missed by this modified STOP-BANG questionnaire (sensitivity $100 \%$ ).

The type of surgery performed, preoperative anesthesia risk class or the duration of general anesthesia were not different in patients with and without OSA. Patients with OSA had significantly higher episodes of apnea or hypopnea and preoperative oxygen desaturation events as diagnosed by the level III sleep study.

On logistic regression, the presence of OSA was independently associated with the development of any predefined PCs by day 7 (OR 3.63, 95\% CI 1.77-7.45, $p<$ 0.01 ) and by day 30 following surgery (OR 3.5, 95\% CI $1.74-7.1, p<0.01)$ and with an increased duration of hospitalization $(p=0.04)$. The proportion of patients with postoperative oxygen desaturation was significantly associated with OSA, regardless of severity of OSA (OR $5.96,95 \%$ CI 2.35-15.1, $p<0.01)$. There was a clear increase in the proportion of patients with respiratory and cardiovascular complications with increasing severity of OSA (Table 2). About $91 \%$ of patients with moderate to severe OSA had at least 1 episode of postoperative oxygen desaturation diagnosed on oximetry.

\section{Discussion}

The magnitude and impact of unrecognized OSA is likely larger in India because of the larger numbers of surgeries performed, the lack of polysomnography services and poor recognition of the syndrome, both among physicians and the general public [18]. Studies suggest that $90 \%$ of patients with OSA remain undiagnosed [2]. Given a prevalence of $2-9.4 \%$ of OSA in our population $[2,19]$, only $8 / 8,824(0.09 \%)$ patients had diagnosed or treated OSA during the screening period in this cohort, suggesting gross underrecognition of OSA. While the actual 
Table 3. Logistic regression of association of unrecognized obstructive sleep apnea (AHI $\geq 5 / \mathrm{h}, n=70 / 182,38.5 \%)$ and postoperative complications

\begin{tabular}{lllcl}
\hline Outcome & Odds ratio & \multicolumn{2}{l}{$95 \%$ confidence interval } & \multirow{2}{*}{$p$ value } \\
& & lower & upper & \\
\hline Any complication at 7 days & 3.63 & 1.77 & 7.45 & $<0.001$ \\
Any complication at 30 days & 3.5 & 1.74 & 7.1 & $<0.001$ \\
Postoperative oxygen desaturation & 5.96 & 1.04 & 3.25 & $<0.001$ \\
\hline
\end{tabular}

prevalence in the original cohort ( 8,824 patients) is unknown, a prevalence of $38.5 \%$ for unrecognized OSA among patients with risk factors is similar to prior studies $[5,6,20,21]$ and points to the magnitude of this problem. Thus, the first recognition of OSA during preoperative evaluation is not an infrequent event.

\section{OSA and Postoperative Complications}

There exists a putative interconnected web of causation in the link between OSA and PCs [22]. Retrospective data suggest that sleep-disordered breathing, REM rebound, sympathetic activation, and episodic hypoxemia all contribute to the development of atrial arrhythmias, pulmonary complications, greater rates of reintubation, and intensive care admission $[9,11]$. However, an independent effect of OSA on PCs has been questioned [23]. While several explanations including ischemic preconditioning or heightened recognition have been proposed, these data are limited by the large proportion of patients with undiagnosed OSA undergoing surgery affecting outcomes. Further, while there is broad consensus on the management of patients with preoperatively diagnosed OSA, there is no consensus on preoperative evaluation for possible undiagnosed OSA.

In a study by Hwang et al. [24], it was noted that the odds of postoperative complications were 7.2 times in a group of subjects with an ODI of $>5 / \mathrm{h}$. Sleep-disordered breathing was assessed based on home oximetry and ODI criteria only. In contrast, the retrospective study by Chong et al. [25] reported no significant increase in PCs in patients managed according to the OSA risk management protocol.

However, we found an independent association of unrecognized OSA with the development of respiratory and cardiovascular PCs (OR 3.5, $p=0.001-0.04$ ) and longer duration of hospital stay, stressing the importance of recognizing OSA in this setting (Table 3). Furthermore, our findings are similar to those reported by Kaw et al. [26] who noted a higher incidence of postoperative hypox- emia (OR, 7.9; $p=0.009$ ), overall complications (OR, 6.9; $p=0.003)$, and a longer hospital length of stay (OR, 1.65; $p=0.049)$.

\section{Preoperative Screening for OSA}

The problem of addressing the impact of undiagnosed OSA on PCs is affected by a lack of information on how to screen and treat OSA without polysomnography preoperatively [27]. Clinical examination has a very poor negative predictive value for OSA [28]. Several screening questionnaires $[12,29]$ have been validated or recommended for preoperative use [7]; however, a positive screen does not imply OSA, questionnaires are not interchangeable and a positive result is not linked to increased 30-day or 1-year mortality [30]. Further, there is little evidence base for the management of patients who score positive on these questionnaires. This lack of clarity has impacted the results of studies on the prophylactic use of continuous airway pressure (CPAP) in patients with OSA to reduce PCs [31, 32].

Questionnaires have to be supplemented by the use of a level III study [33] or postoperative respiratory monitoring [4] to identify OSA. The use of a level III study in patients being at risk for OSA is needed for reliably ruling out OSA preoperatively and can establish a definite intraand postoperative plan. Further, sleep-disordered breathing as assessed by sleep studies has been associated independently with the above-mentioned predefined complications [24].

The STOP-BANG questionnaire is the most widely used validated preoperative OSA questionnaire [12]. It was validated in Caucasians and it is likely that the age, BMI and neck circumference cutoffs are very different in other populations. However, no data exist on such cutoffs and the unmodified STOP-BANG questionnaire continues to be used extensively, despite such concerns [34]. Our data suggest that cutoffs based on Caucasian morphology have poor sensitivity in South Asians. 
The questionnaire both under- (17\%) and overdiagnosed (28\%) OSA in the study population when compared with the level III sleep study. Given the poor performance of the STOP-BANG questionnaire, a preoperative level III study in patients with $\geq 2$ risk factors for OSA would be the appropriate method for the evaluation of undiagnosed OSA preoperatively.

Trials of continuous CPAP in the postoperative period for OSA have been negative $[31,32]$. This could be due to a false association of PCs with OSA in retrospective studies or due to failure in excluding OSA due to limited sensitivity of clinical screening in trials of CPAP [35].

The strengths of our study are a statistically well-powered and prospective design and a follow-up with clearly defined PCs. The limitations include the small number of patients with moderate to severe OSA (6\%) and absence of polysomnography for confirmation of OSA. Polysomnography is unlikely to be accessible to patients presenting during preoperative evaluation and our design was based on this pragmatic understanding.

\section{Conclusions}

Unrecognized OSA remains very common during preoperative evaluation of surgical patients. Unrecognized OSA is independently associated with the development of postoperative oxygen desaturation and development of
PCs by day 7 and 30 . The STOP-BANG questionnaire has poor sensitivity in South Indians. The level III sleep study in patients with $\geq 2$ risk factors for OSA can help identify undiagnosed OSA and prevent anticipated PCs in OSA.

\section{Acknowledgement}

St. John's Research Institute supported the present article.

\section{Financial Disclosure and Conflicts of Interest}

The authors declare that there is no conflict of interest.

\section{Author Contributions}

Concept, design, screening of patients: U.D., S.R., P.R., and P.J.D. Selection and recruitment of study subjects: U.D., S.R., A.K., and P.R. Informed consent, laboratory investigations: U.D., S.R., A.K., and P.R. Laboratory report interpretation: U.D. Treatment decision: S.R., A.K., and P.R. Patient/subject care during study period: U.D., A.K., P.R., and G.A.D. SAE evaluation and reporting: U.D., A.K., P.R., and G.A.D. Examination of patients on follow-up: U.D., P.R., and G.A.D. Data collection and monitoring of data: A.K. Interpretation of data, statistical analysis and interpretation: U.D., S.R., P.R., and P.J.D. Maintaining patients file and master file of project: U.D. Drafting final report: U.D., S.R., and P.J.D. Publication: U.D., S.R., A.K., P.R., and G.A.D.

\section{References}

1 Weiser TG, Regenbogen SE, Thompson KD, Haynes AB, Lipsitz SR, Berry WR, et al: An estimation of the global volume of surgery: a modelling strategy based on available data. Lancet 2008;372:139-144.

2 Reddy EV, Kadhiravan T, Mishra HK, Sreenivas V, Handa KK, Sinha S, et al: Prevalence and risk factors of obstructive sleep apnea among middle-aged urban Indians: a community-based study. Sleep Med 2009;10:913918.

3 Udwadia ZF, Doshi AV, Lonkar SG, Singh CI: Prevalence of sleep-disordered breathing and sleep apnea in middle-aged urban Indian men. Am J Respir Crit Care Med 2004;169: 168-173.

4 Blake DW, Chia PH, Donnan G, Williams DL: Preoperative assessment for obstructive sleep apnoea and the prediction of postoperative respiratory obstruction and hypoxaemia. Anaesth Intensive Care 2008;36:379-384.
5 Fidan H, Fidan F, Unlu M, Ela Y, Ibis A, Tetik L: Prevalence of sleep apnoea in patients undergoing operation. Sleep Breath 2006;10: 161-165.

6 Finkel KJ, Searleman AC, Tymkew H, Tanaka CY, Saager L, Safer-Zadeh E, et al: Prevalence of undiagnosed obstructive sleep apnea among adult surgical patients in an academic medical center. Sleep Med 2009;10:753-758.

7 American Society of Anesthesiologists Task Force on Perioperative Management of Patients with Obstructive Sleep Apnea: Practice guidelines for the perioperative management of patients with obstructive sleep apnea: an updated report by the American Society of Anesthesiologists Task Force on Perioperative Management of patients with obstructive sleep apnea. Anesthesiology 2014;120:268286.

8 Hanly P, Sasson Z, Zuberi N, Lunn K: ST-segment depression during sleep in obstructive sleep apnea. Am J Cardiol 1993;71:13411345.
9 Memtsoudis SG, Stundner O, Rasul R, Chiu YL, Sun X, Ramachandran SK, et al: The impact of sleep apnea on postoperative utilization of resources and adverse outcomes. Anesth Analg 2014;118:407-418.

10 Mokhlesi B, Hovda MD, Vekhter B, Arora VM, Chung F, Meltzer DO: Sleep-disordered breathing and postoperative outcomes after bariatric surgery: analysis of the nationwide inpatient sample. Obes Surg 2013;23:18421851.

11 Mokhlesi B, Hovda MD, Vekhter B, Arora VM, Chung F, Meltzer DO: Sleep-disordered breathing and postoperative outcomes after elective surgery: analysis of the nationwide inpatient sample. Chest 2013;144:903-914.

12 Chung F, Yegneswaran B, Liao P, Chung SA, Vairavanathan S, Islam S, et al: STOP questionnaire: a tool to screen patients for obstructive sleep apnea. Anesthesiology 2008; 108:812-821.
Devaraj/Rajagopala/Kumar/ Ramachandran/Devereaux/D'Souza 
13 Stegenga $\mathrm{H}$, Haines A, Jones $\mathrm{K}$, Wilding J; Guideline Development Group: Identification, assessment, and management of overweight and obesity: summary of updated NICE guidance. BMJ 2014;349:g6608.

14 WHO Expert Consultation: Appropriate body-mass index for Asian populations and its implications for policy and intervention strategies. Lancet 2004;363:157-163.

15 El Shayeb M, Stafinski T, Pawluk L, Menon D: L-ATMLS. Diagnostic accuracy of level 3 portable sleep tests versus level 1 polysomnography for sleep-disordered breathing: a systematic review and meta-analysis. CMAJ 2014; 186:E25-E51.

16 Kim SE, Park BS, Park SH, Shin KJ, Ha SY, Park JS, Park KM: Predictors for presence and severity of obstructive sleep apnea in snoring patients: significance of neck circumference. J Sleep Med 2015;12:34-38.

17 Kang HH, Kang JY, Ha JH, Lee J, Kim SK, Moon HS, Lee SH: The associations between anthropometric indices and obstructive sleep apnea in a Korean population. PLoS One 2014;9:12.

18 Sharma SK, Ahluwalia G: Epidemiology of adult obstructive sleep apnoea syndrome in India. Indian J Med Res 2010;131:171-175.

19 Udwadia ZF, Doshi AV, Lonkar SG, Singh CI: Prevalence of sleep disordered breathing and sleep apnoea in middle-aged urban Indian men. Am J Respir Crit Care Med 2004;169: 168-173.

20 Agrawal S, Gupta R, Lahan V, Mustafa G, Kaur U: Prevalence of obstructive sleep apnea in surgical patients presenting to a tertiary care teaching hospital in India: a preliminary study. Saudi J Anaesth 2013;7:155-159.
21 Pizarro C, Schaefer C, Kimeu I, Pingel S, Horlbeck F, Tuleta I, et al: Underdiagnosis of obstructive sleep apnoea in peripheral arterial disease. Respiration 2015;89:214-220.

22 Adesanya AO, Lee W, Greilich NB, Joshi GP: Perioperative management of obstructive sleep apnea. Chest 2010;138:1489-1498.

23 Blake DW, Donnan G: Preoperative screening for obstructive sleep apnoea - are we losing sleep over nothing? Anaesth Intensive Care 2010;38:210-211.

24 Hwang D, Shakir N, Limann B, Sison C, Kalra S, Shulman L, et al: Association of sleep-disordered breathing with postoperative complications. Chest 2008;133:1128-1134.

25 Chong CT, Tey J, Leow SL, Low W, Kwan $\mathrm{KM}$, Wong YL, et al: Management plan to reduce risks in perioperative care of patients with obstructive sleep apnoea averts the need for presurgical polysomnography. Ann Acad Med Singapore 2013;42:110-119.

26 Kaw R, Pasupuleti V, Walker E, Ramaswamy A, Foldvary-Schafer N: Postoperative complications in patients with obstructive sleep apnea. Chest 2012;141:436-441.

27 Ramachandran SK, Josephs LA: A meta-analysis of clinical screening tests for obstructive sleep apnea. Anesthesiology 2009;110:928939.

28 Sareli AE, Cantor CR, Williams NN, Korus G, Raper SE, Pien G, et al: Obstructive sleep apnea in patients undergoing bariatric surgery - a tertiary center experience. Obes Surg 2011;21:316-327.
29 Chung F, Ward B, Ho J, Yuan H, Kayumov L, Shapiro C: Preoperative identification of sleep apnea risk in elective surgical patients, using the Berlin questionnaire. J Clin Anesth 2007; 19:130-134.

30 Lockhart EM, Willingham MD, Abdallah AB, Helsten DL, Bedair BA, Thomas J, et al: Obstructive sleep apnea screening and postoperative mortality in a large surgical cohort. Sleep Med 2013;14:407-415.

31 Liao P, Luo Q, Elsaid H, Kang W, Shapiro CM, Chung F: Perioperative auto-titrated continuous positive airway pressure treatment in surgical patients with obstructive sleep apnea: a randomized controlled trial. Anesthesiology 2013;119:837-847.

32 O'Gorman SM, Gay PC, Morgenthaler TI: Does autotitrating positive airway pressure therapy improve postoperative outcome in patients at risk for obstructive sleep apnea syndrome? A randomized controlled clinical trial. Chest 2013;144:72-78.

33 Pereira EJ, Driver HS, Stewart SC, Fitzpatrick MF: Comparing a combination of validated questionnaires and level III portable monitor with polysomnography to diagnose and exclude sleep apnea. J Clin Sleep Med 2013;9: 1259-1266.

34 Corso RM, Cattano D: Does STOP-BANG really predict postoperative critical care admission? Anaesthesia 2013;68:1200.

35 Nugent KM, Phy M, Raj R: Obstructive sleep apnea and post-operative complications: single center data, review of literature and guidelines for practicing internists and surgeons. Surg Scis 2012;2012:65-71. 